\title{
Co-Occurrence of NDM-9 and MCR-I in a Human Gut Colonized Escherichia coli STIOII
}

\author{
Ganfeng Liang ${ }^{1} * *$ \\ Yuting Rao ${ }^{2,3, *}$ \\ Shuang Wang ${ }^{4}$ \\ Xiaohui $\mathrm{Chi}^{2}$ \\ $\mathrm{Hao} \mathrm{Xu}^{2}$ \\ Yang Shen' \\ 'Department of Infectious Diseases, The \\ First Hospital of Taizhou, Taizhou, \\ People's Republic of China; \\ ${ }^{2}$ Collaborative Innovation Center for \\ Diagnosis and Treatment of Infectious \\ Diseases, State Key Laboratory for \\ Diagnosis and Treatment of Infectious \\ Diseases, The First Affiliated Hospital, \\ College of Medicine, Zhejiang University, \\ Hangzhou, People's Republic of China; \\ ${ }^{3}$ Department of Laboratory Medicine, \\ Puyang Oil Field General Hospital, \\ Puyang, People's Republic of China; \\ ${ }^{4}$ Institute of Bacterial Infection Disease \\ Control, Shandong Centre for Disease \\ Control and Prevention, Jinan, People's \\ Republic of China
}

*These authors contributed equally to this work
Correspondence: Yang Shen; Hao Xu Email Shenyanghtt@163.com; xuhao0523@zju.edu.cn
Background: The emergence of the plasmid-borne colistin-resistant gene ( $m c r-1)$ poses a great threat to human health. What is worse, the recent observations of the coexistence of $m c r-1$ with carbapenemase encoding genes in some bacteria caused even more concern. Yet, there is a lack of observations of such strains in the human gut.

Methods: The isolation of E. coli L889 was performed on selective medium plates. Antibiotic susceptibilities were determined by an agar dilution and a broth microdilution method. Multi-locus sequence typing (MLST) and acquired resistance genes were also characterized. Transferability of $b l a_{\mathrm{NDM}-9} / \mathrm{mcr}$ - 1 -carrying plasmids was determined by conjugation, replicon typing and S1-Pulsed-field gel electrophoresis (S1-PFGE), and Southern blotting. The sequences of these plasmids were analyzed by using whole-genome sequencing with Illumina Novaseq and Nanopore platforms.

Results: $E$. coli L889 was identified as ST1101 concomitantly carrying bla NDM-9 $_{\text {and }} m c r-1$ from a stool sample. Antimicrobial susceptibility tests showed that it was resistant to various antimicrobial agents and only susceptible to tigecycline. Notably, $b l a_{\mathrm{NDM}-9}$ was located on a $\sim 114-\mathrm{kb}$ untypable plasmid, while $m c r-1$ was located on a $\sim 63-\mathrm{kb}$ IncI2 plasmid.

Conclusion: Our research, to our knowledge, first reported an ST1101 E. coli strain with an untypeable $b l a_{\mathrm{NDM}-9}$-harbouring plasmid and an IncI2 mcr-1-carrying plasmid. The colonized $E$. coli strains potentially contribute to the dissemination and transfer of $b l a_{\mathrm{NDM}-9}$ and mcr-1 to clinical isolates, which is a considerable threat to public health and should be closely monitored.

Keywords: bla $a_{\mathrm{NDM}-9}$, mcr-1, gut, Escherichia coli, ST1101

\section{Background}

The global dissemination of colistin resistance, due to transferable $m c r$-genes, threatens public and animal health as there are limited therapeutic options. Since the plasmid-mediated colistin resistance gene $m c r-1$ was first reported in Escherichia coli isolates in China, several reports confirmed that $m c r-1$ has spread in several Enterobacteriaceae species on different continents and from various samples. ${ }^{1-7}$ Of great clinical concern are the inevitable co-occurrence of $\mathrm{mcr}$-genes and carbapenemresistance genes among Enterobacteriaceae and the widespread resistance genes in the environment, which eventually aggravate the selection process in the occurrence of true pan-drug resistance. ${ }^{1,8,9}$

The first NDM-9-producing K. pneumoniae strain was isolated in 2014 in China, which showed that the bla $a_{\mathrm{NDM}-9}$ gene encodes a protein with one amino acid substitution (E152K) compared with NDM-1. ${ }^{10}$ Subsequently, the NDM-9 variant was sporadically reported in Asian and European countries. ${ }^{11-13}$ It is worthy to note 
that NDM-9 presented more significant enzyme activity than NDM-1 on all tested $\beta$-lactams except monobactams, slightly higher hydrolytic activity for cefotaxime, cefoxitin, imipenem, and meropenem, and higher affinity for imipenem and meropenem. ${ }^{11}$

Previously we conducted a prospective, observational cohort study involving inpatients to screen carbapenemase-producing Enterobacteriaceae (CPE) from stool samples. ${ }^{14}$ In this work, we report the isolation of coproducing of MCR-1 and NDM-9 in a human gut colonized $E$. coli L889 from that follow-up investigation. We also described the antimicrobial susceptibility profile and plasmid characteristics of this isolate.

\section{Materials and Methods}

\section{Bacterial Isolation and Susceptibility} Testing

Previously, we sampled 811 nonduplicate stool samples from 443 inpatients and screened for carbapenemaseproducing Enterobacteriaceae isolates. ${ }^{14}$ The E. coli L889 strain was isolated from a fecal sample of a 21-years old male patient admitted with abdominal pain. Bacterial identification was confirmed by matrix-assist laser desorption ionization time-of-flight mass spectrometry (MALDI-TOF -MS) (Bruker, Bremen, Germany). The carbapenemase encoding gene (bla $\left.a_{\mathrm{NDM}}\right)$ was identified using PCR (Primer: Forward-ATGGAATTGCCCAATATTATGCAC, Reverse-TCAGCGCAGCTTGTCGGC), and DNA sequencing was performed on the PCR positive isolates using Sanger sequencing. Antimicrobial susceptibility testing (AST) was conducted by agar dilution and broth microdilution, using Escherichia coli (ATCC 25922) as the control. AST results were interpreted following the Clinical and Laboratory Standards Institute (CLSI) 2020 standards. ${ }^{15}$

\section{Whole-Genome Sequencing and in silico Analysis}

Whole-genome sequencing (WGS) of L889 was performed using Illumina NovaSeq 6000 (Illumina, USA) and Nanopore (Oxford Nanotechnology, UK) platforms in Novogene (Beijing, China). The hybrid assembly of Illumina and Nanopore reads was performed using Unicycler 20 (v0.4.7). PlasmidFinder (https://cge.cbs.dtu. dk/services/PlasmidFinder/) and Resfinder ${ }^{16}$ were used to determine the plasmid replicon type and acquired resistance genes. Genotyping was performed to query the seven via the multi-locus sequence typing MLST web service (https://cge.cbs.dtu.dk/services/MLST/).

\section{Plasmid Analysis and Conjugation Assays}

The number and size of the plasmid of the strains were characterized by S1-PFGE. The location of $b{ }_{\text {NDM-9 }}$ and $m c r-1$ genes was confirmed by Southern blotting and hybridization with a digoxigenin-labeled $b l a_{\mathrm{NDM}-9}$ and $m c r-1$ probe using DIG-High Prime DNA Labeling and Detection Starter Kit II (Roche Diagnostics). Conjugation transfer experiments were conducted to explore the transferability of plasmids with rifampicin-resistant $E$. coli 600 as the recipient strain as recipients, as described previously. ${ }^{17}$ After that, using Mueller-Hinton agar (OXOID, Hampshire, United Kingdom) plates that contained both $200 \mathrm{mg} / \mathrm{L}$ rifampicin (Meilunbio, Dalian, China) and $2 \mathrm{mg} / \mathrm{L}$ meropenem to select $b l a_{\mathrm{NDM}-9}$ carrying transconjugants, and $200 \mathrm{mg} / \mathrm{L}$ rifampicin with $2 \mathrm{mg} / \mathrm{L}$ colistin to select $m c r-1$ carrying transconjugants, respectively. The final identification of transconjugants, including MALDI-TOF/MS identification, resistance genes detection, and AST, to confirm whether the experiments succeed.

\section{Results and Discussion}

E. coli L889 was isolated from a 21-years old patient on November 2017. Antimicrobial susceptibility testing by showed that L889 was resistant to various types of antimicrobial agents, including amoxicillin/clavulanate (MIC $=32 \mu \mathrm{g} / \mathrm{mL}$ ), piperacillin/tazobactam (MIC > $128 \mu \mathrm{g} /$ $\mathrm{mL}$ ), cefotaxime (MIC $>128 \mu \mathrm{g} / \mathrm{mL}$ ), ceftazidime (MIC $>128 \mu \mathrm{g} / \mathrm{mL}$ ), cefpirome (MIC $>128 \mu \mathrm{g} / \mathrm{mL}$ ), cefepime $(\mathrm{MIC}=128 \mu \mathrm{g} / \mathrm{mL})$, meropenem $(\mathrm{MIC}=4 \mu \mathrm{g} / \mathrm{mL})$, ertapenem $(\mathrm{MIC}=16 \mu \mathrm{g} / \mathrm{mL})$, imipenem $(\mathrm{MIC}=8 \mu \mathrm{g} /$ $\mathrm{mL}$ ), aztreonam $(\mathrm{MIC}=128 \mu \mathrm{g} / \mathrm{mL})$, amikacin $(\mathrm{MIC}>$ $128 \mu \mathrm{g} / \mathrm{mL}$ ), gentamicin (MIC $\geq 128 \mu \mathrm{g} / \mathrm{mL}$ ), levofloxacin $(\mathrm{MIC}=32 \mu \mathrm{g} / \mathrm{mL}$ ), ciprofloxacin $(\mathrm{MIC}>64 \mu \mathrm{g} /$ $\mathrm{mL}$ ), trimethoprim/sulfamethoxazole (MIC $>8 \mu \mathrm{g} / \mathrm{mL}$ ), tetracycline ( $\mathrm{MIC}=64 \mu \mathrm{g} / \mathrm{mL})$, fosfomycin ( $\mathrm{MIC}>512$ $\mu \mathrm{g} / \mathrm{mL}$ ), and colistin ( $\mathrm{MIC}=16 \mu \mathrm{g} / \mathrm{mL}$ ). It only susceptible to tigecycline with the MIC value of $0.125 \mu \mathrm{g} / \mathrm{mL}$.

Analyzing the genome sequence of L889 by MLST 2.0 showed that it belonged to ST-1011. It is worthy to note that NDM-producing E. coli ST-1011 is prevalent in duck farms in southeast coastal China. ${ }^{18}$ A previous study conducted in Lebanon found that ST1011 was one of the most widely identified clones associated with $m c r-1$-carrying E. coli and to the poultry sector. Our work further highlights the cooccurrence of NDM-9 and MCR-1 in E. coli ST1011 from 
human gut colonized isolate. The dissemination of $E$. coli ST1011 from the poultry sector to the human sector indicated that we should closely monitor this clone among clinical settings.

A total of 26 acquired resistance genes were predicted from the genome sequence of L889 by ResFinder. These ARGs enabled L889 to exhibit resistance to different types of antimicrobial agents, including beta-lactams (bla $a_{\mathrm{CTX}-\mathrm{M}-123}, \quad b l a_{\mathrm{NDM}-9}, \quad b l a_{\mathrm{CT}-\mathrm{M}-164}, \quad b l a_{\mathrm{TEM}-1 \mathrm{~B}}$, and $\left.b l a_{\mathrm{OXA}-1}\right)$, fosfomycin (fos $\left.A 3\right)$, colistin ( $\left.m c r-1\right)$, sulphonamide (sul and sul2), phenicol (floR), aminoglycosides (aadA2, aadA22, aac(3)-Iid, aac6'-Ib-cr, aph3'"-Ib, aph6$I d$, $\operatorname{arm} A$, and $\left.a p h 3^{\prime}-I a\right)$, macrolide ( $m d f A$ and $\left.m p h(A)\right)$, quinolone (oqxA and oqxB), tetracycline $(\operatorname{tet}(A))$, chloramphenicol (catB4), rifampin (ARR-3), and trimethoprim (dfrA12 and dfrA17). To a further extent, the cooccurrence of $m c r-1$ and $b l a_{\mathrm{NDM}}$ genes in $E$. coli has been occasionally detected in human and animal sectors. ${ }^{6,19-22}$
As far as we know, L889 is the first reported gut-originated E. coli strain that harbored both $m c r-1$ and $b l a_{\mathrm{NDM}-9}$ genes. S1-PFGE revealed that L889 carried four plasmids (Figure 1). Further investigation by Plasmidfinder and Blastn confirmed that $m c r-1$ carried by a $63 \mathrm{kp}$ plasmid (pL889-MCR1), and bla $a_{\text {NDM-9 }}$ encoded by a 114 bp plasmid (pL889-NDM9). Plasmids were sequenced on Illumina and Nanopore platforms. Two complete plasmids were assembled by the sequencing reads from both two platforms. The complete sequences of these two plasmids were deposited in The National Center for Biotechnology Information with the accession numbers of MZ062604 (pL889-MCR1) and MZ062605 (pL889-NDM9).

Plasmid pL889-NDM9 is a 114,985 bp circular untypeable plasmid. Nucleotide sequence alignment revealed that the backbone of pL889-NDM9 exhibited high similarity to plasmid pHNTH02-1 (MG196294), which was previously recovered from E. coli THSJ02 from retail chicken meat in

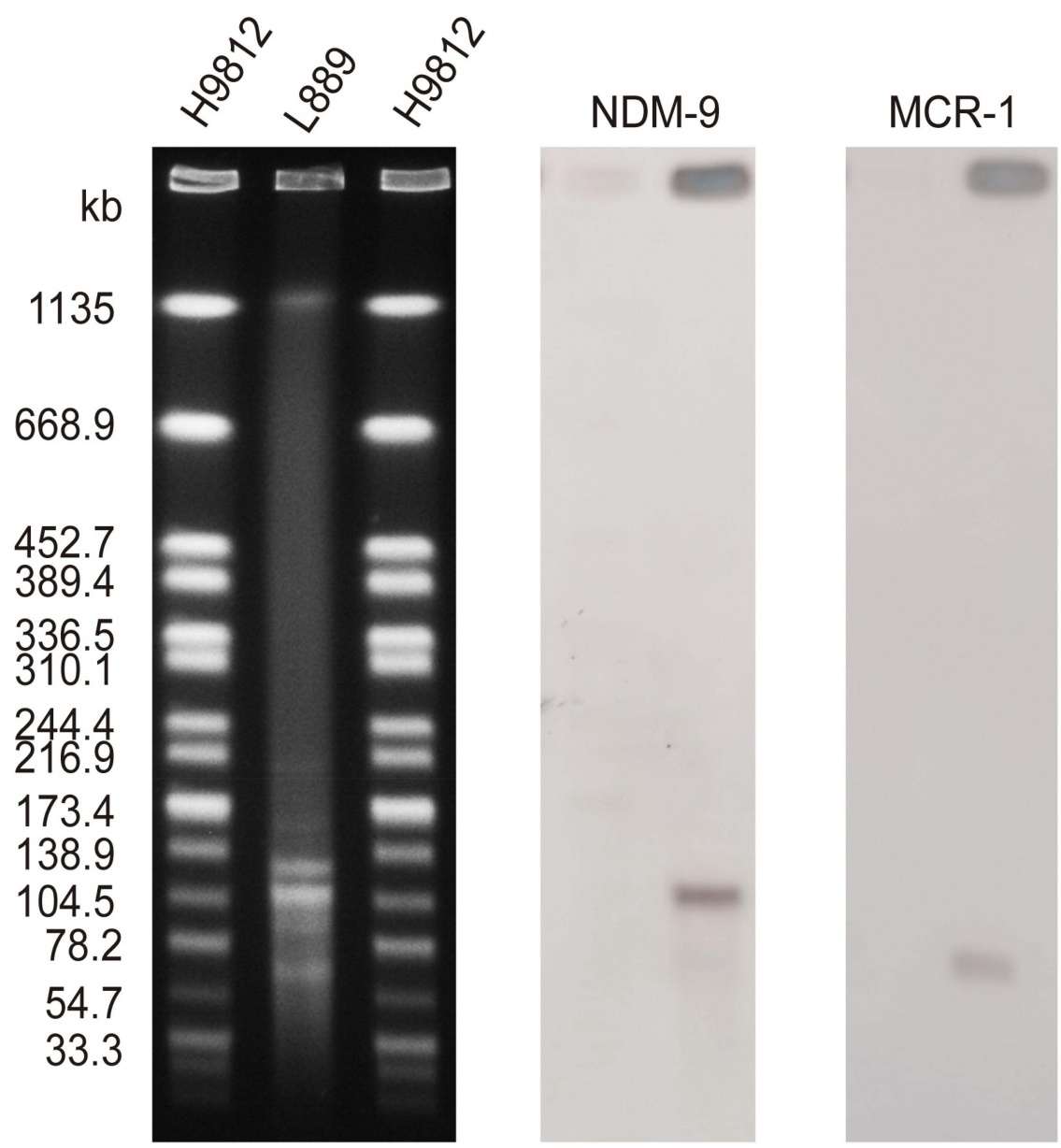

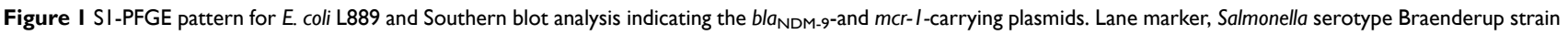
H98I2 as a reference size standard; L889, PFGE result of SI-digested plasmid DNA of strain E. coli L889; NDM-9, Southern blotting of L889 with the probes specific to the bla $a_{\text {NDM-9; }}$ MCR-I, Southern blotting of L889 with the probes specific to the mcr-I. 
Guangzhou, China, ${ }^{23}$ possesses $99 \%$ coverage and $99.62 \%$ identity (Figure 2A). pL889-NDM9 also showed highly homologous to pEC013 (MG545909), pNDM-T2 (MN335919) and pHNSD138-1 (MG271839), which were all found in E. coli isolated from chicken samples
(83-90\% coverage and $99.14-99.66 \%$ identity). Furthermore, genetic environment characterization revealed that $b l a_{\mathrm{NDM}-9}$ was located in an ISCR1 complex class 1 integron with two copies of IS26, with a conserved structure of IS26- $\Delta \mathrm{IS} A$ ba 125-bla $a_{\mathrm{NDM}-9}-$ ble $_{\mathrm{MBL}}-t r p F-t a t$
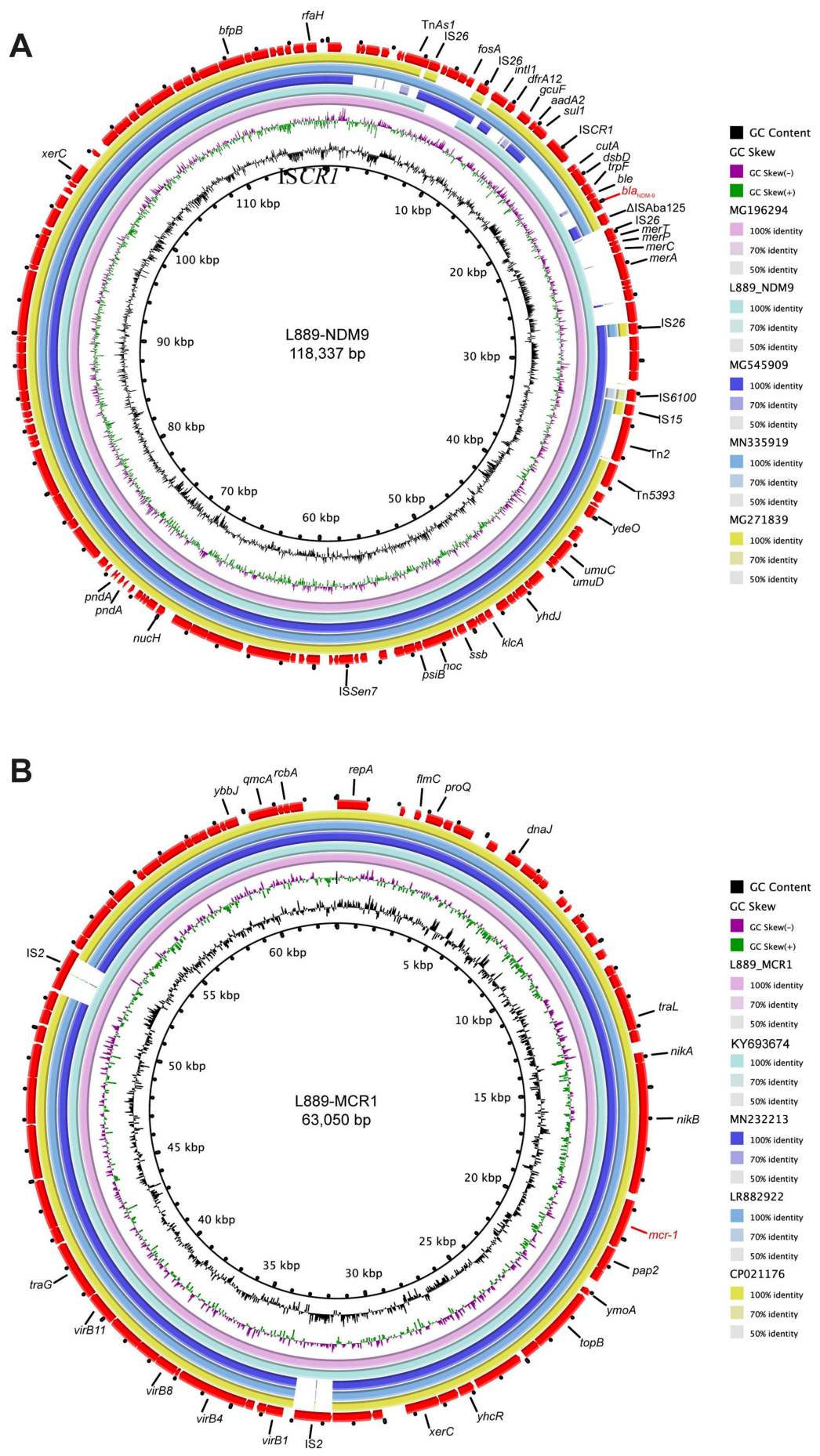

Figure 2 Comparative analysis of plasmids pL889-NDM9 and pL889-MCRI detected in E. coli L889. (A) Comparison of bla NDM-9 coding region of plasmid pL889-NDM9 with plasmid pHNTH02-I (MG196294), pEC0I3 (MG545909), pNDM-T2 (MN335919), and pHNSDI38-I (MG27I839). (B) Comparison of mcr-I-carrying plasmid pL889MCRI with plasmids pHNTH02-I (KY693674), pHLJI79-34 (MN2322I3), and p5CRE5I-MCR-I (CP02II76). The circular map was generated with the BLAST Ring Image Generator (http://brig.sourceforge.net/). 
Table I Minimum Inhibitory Concentrations (MICs) of Tested Antibiotics for the bla NDM-9- and Mcr-I- Positive Escherichia coli STIIOI Strain and Transconjugants

\begin{tabular}{|l|l|l|l|}
\hline Agents & E. coli L889 & Transconjugant (NDM-9) & Transconjugant (MCR-I) \\
\hline Amoxicillin/clavulanate & $32(\mathrm{R})$ & $32(\mathrm{R})$ & $64(\mathrm{R})$ \\
Piperacillin/tazobactam & $>128(\mathrm{R})$ & $>128(\mathrm{R})$ & $>128(\mathrm{R})$ \\
Cefotaxime & $>128(\mathrm{R})$ & $>128(\mathrm{R})$ & $>128(\mathrm{R})$ \\
Ceftazidime & $>128(\mathrm{R})$ & $>128(\mathrm{R})$ & $>128(\mathrm{R})$ \\
Cefpirome & $>128(\mathrm{R})$ & $64(\mathrm{R})$ & $>128(\mathrm{R})$ \\
Cefepime & $\mathrm{I} 28(\mathrm{R})$ & $64(\mathrm{R})$ & $128(\mathrm{R})$ \\
Meropenem & $4(\mathrm{R})$ & $2(\mathrm{l})$ & $2(\mathrm{I})$ \\
Imipenem & $8(\mathrm{R})$ & $8(\mathrm{R})$ & $4(\mathrm{R})$ \\
Ertapenem & $\mathrm{I}(\mathrm{R})$ & $8(\mathrm{R})$ & $2(\mathrm{~S})$ \\
Aztreonam & $\mathrm{I} 28(\mathrm{R})$ & $0.5(\mathrm{~S})$ & $128(\mathrm{R})$ \\
Gentamicin & $>128(\mathrm{R})$ & $\mathrm{I}(\mathrm{S})$ & $>128(\mathrm{R})$ \\
Amikacin & $>128(\mathrm{R})$ & $4(\mathrm{~S})$ & $>128(\mathrm{R})$ \\
Levofloxacin & $32(\mathrm{R})$ & $0.5(\mathrm{~S})$ & $64(\mathrm{R})$ \\
Ciprofloxacin & $>64(\mathrm{R})$ & $0.5(\mathrm{~S})$ & $>64(\mathrm{R})$ \\
Trimethoprim/sulfamethoxazole & $>8(\mathrm{R})$ & $<0.125(\mathrm{~S})$ & $>8(\mathrm{R})$ \\
Tetracycline & $64(\mathrm{R})$ & $0.5(\mathrm{~S})$ & $64(\mathrm{R})$ \\
Fosfomycin & $>5 I 2(\mathrm{R})$ & $\mathrm{I}(\mathrm{S})$ & $>512(\mathrm{R})$ \\
Nitrofurantoin & $64(\mathrm{I})$ & $4(\mathrm{~S})$ & $16(\mathrm{~S})$ \\
Tigecycline & $0.125(\mathrm{~S})$ & $0.25(\mathrm{~S})$ & $0.125(\mathrm{~S})$ \\
Colistin & $\mathrm{I}(\mathrm{R})$ & $2(\mathrm{~S})$ & $16(\mathrm{R})$ \\
\hline
\end{tabular}

- $\triangle$ catA-ISCR1-qacED1-sul1-aad2-gcuF-dfrA12-intI1-IS 26, which is consistent with the plasmid pHNTH02-1. Our results further supported that this conserved structure may be associated with the transfer and spread of NDM-9-carrying plasmids. ${ }^{23}$ Moreover, an antimicrobial resistance gene conferring resistance to fosfomycin, fos $A 3$, is located upstream of NDM-9 region. To date, the emergence of bla $_{\text {NDM-9 }}$ has been reported in E. coli, ${ }^{24,25}$ Klebsiella pneumoniae, ${ }^{10}$ Klebsiella variicola, ${ }^{11}$ and Cronobacter sakazakii. $^{26}$ Our discovery of this plasmid supplemented previous studies and further highlighted the dissemination of bla $a_{\text {NDM-9 }}$ gene-carrying plasmids in human gut colonized isolates.

Plasmid pL889-MCR1 is a 63,050 bp circular plasmid belonging to IncI2, which was associated with the global dissemination of MCR-1-producing E. coli from animal and human sectors. ${ }^{27-31}$ Nucleotide sequence alignment indicated that it aligned very well to plasmids pHNTH02-1 (KY693674), pHLJ179-34 (MN232213), and p5CRE51-MCR-1 (CP021176) (Figure 2B). Of note, p5CRE51-MCR-1 carrying isolate was recovered from a urinary tract infection, which represents the first case reported an E. coli strain co-producing MCR-1 and NDM9. ${ }^{32}$ Annotation of the plasmid sequence revealed a typical structure surrounding the mcr-1 gene (nikA-nikB-mcr -1-pap2) in pL889-MCR1. Interestingly, this conserved structure was popularly identified in clinical and animal isolates. $^{28,33}$

Conjugation analysis confirmed that both $b l a_{\mathrm{NDM}-9}$ and mcr-1 genes were transferable to the recipient cells (Table 1). These data are consistent with previous investigations that MCR-1- or NDM-9-positive Enterobacteriaceae exhibit in vitro antibiotic resistance against most antimicrobial agents. ${ }^{23-26,32}$ It is worthy to note that tigecycline showing well in vitro activity against these bacteria carrying MCR-1 and NDM-9 in the current case and previous investigations, ${ }^{24,32}$ a further large-scale study to evaluate the activity of tigecycline $s$ is warranted. ${ }^{32}$

Collectively, the present case reported the complete sequences of an IncI 2 type $m c r-1$ carrying plasmid and an untypeable type $b l a_{\text {NDM-9 }}$ carrying plasmid in an $E$. coli isolated from a stool sample. Furthermore, our data also clearly demonstrated that colonized E. coli strains potentially contribute to the dissemination and transfer of $b l a_{\mathrm{NDM}-9}$ and $m c r-1$ to clinical isolates. To lower the risk of disseminating this multidrug-resistant strain in stool samples, closely monitoring is needed in the future. 


\section{Acknowledgments}

This work was supported by the National Key Research and Development Program of China (No. 2016YFD0501105), the National Natural Science Foundation of China (No. 82072314), and Shandong Provincial Medical Science and Technology Development Project (no.2017WS447).

\section{Disclosure}

The authors report no conflicts of interest in this work.

\section{References}

1. Zheng B, Dong H, Xu H, et al. Coexistence of MCR-1 and NDM-1 in clinical Escherichia coli isolates. Clin Infect Dis. 2016;63 (10):1393-1395. doi:10.1093/cid/ciw553

2. Zheng B, Xu H, Yu X, et al. Low prevalence of MCR-1-producing Klebsiella pneumoniae in bloodstream infections in China. Clin Microbiol Infect. 2018;24(2):205-206. doi:10.1016/j.cmi.2017.08.004

3. Zheng B, Feng Y. MCR-1-producing Salmonella Typhimurium ST34 links animal foods to human community infections. EBioMedicine. 2019;42:10-11. doi:10.1016/j.ebiom.2019.03.073

4. Ji X, Zheng B, Berglund $B$, et al. Dissemination of extended-spectrum beta-lactamase-producing Escherichia coli carrying mcr-1 among multiple environmental sources in rural China and associated risk to human health. Environ Pollut. 2019;251:619-627. doi:10.1016/j.envpol.2019.05.002

5. Zheng B, Huang C, Xu H, et al. Occurrence and genomic characterization of ESBL-producing, MCR-1-harboring Escherichia coli in farming soil. Front Microbiol. 2017;8:2510. doi:10.3389/ fmicb.2017.02510

6. Zheng B, Lv T, Xu H, et al. Discovery and characterisation of an escherichia coli ST206 strain producing NDM-5 and MCR-1 from a patient with acute diarrhoea in China. Int $J$ Antimicrob Agents. 2018;51(2):273-275. doi:10.1016/j.ijantimicag.2017.09.005

7. Sun J, Zhang H, Liu YH, Feng Y. Towards understanding MCR-like colistin resistance. Trends Microbiol. 2018;26(9):794-808. doi:10.1016/j.tim.2018.02.006

8. Shen Z, Hu Y, Sun Q, et al. Emerging carriage of NDM-5 and MCR-1 in escherichia coli from healthy people in multiple regions in China: a Cross Sectional Observational Study. EClinicalMedicine. 2018;6:11-20. doi:10.1016/j.eclinm.2018.11.003

9. Khan H, Miao X, Liu M, Ahmad S, Bai X. Behavior of last resort antibiotic resistance genes (mcr-1 and blaNDM-1) in a drinking water supply system and their possible acquisition by the mouse gut flora. Environ Pollut. 2020;259:113818. doi:10.1016/j.envpol.2019.113818

10. Wang X, Li H, Zhao C, et al. Novel NDM-9 metallo-beta-lactamase identified from a ST107 Klebsiella pneumoniae strain isolated in China. Int J Antimicrob Agents. 2014;44(1):90-91. doi:10.1016/j. ijantimicag.2014.04.010

11. Di DY, Jang J, Unno T, Hur HG. Emergence of Klebsiella variicola positive for NDM-9, a variant of New Delhi metallo-beta-lactamase, in an urban river in South Korea. $J$ Antimicrob Chemother. 2017;72:1063-1067.

12. Nuesch-Inderbinen M, Zurfluh K, Stevens MJA, Stephan R. Complete and assembled genome sequence of an NDM-9- and CTX-M-15-producing Klebsiella pneumoniae ST147 wastewater isolate from Switzerland. J Glob Antimicrob Resist. 2018;13:53-54. doi:10.1016/j.jgar.2018.03.001

13. Falcone M, Giordano C, Barnini S, et al. Extremely drug-resistant NDM-9-producing ST147 Klebsiella pneumoniae causing infections in Italy, May 2020. Euro Surveill. 2020;25(48):2001779. doi:10.2807/1560-7917.ES.2020.25.48.2001779
14. Zheng B, Xu H, Lv T, et al. Stool samples of acute diarrhea inpatients as a reservoir of ST11 hypervirulent KPC-2-producing Klebsiella pneumoniae. mSystems. 2020;5(3):e00498-20. doi:10.1128/ mSystems.00498-20

15. Nair S, Unnikrishnan M, Turner K, et al. Molecular analysis of fluoroquinolone-resistant salmonella paratyphi A isolate, India. Emerg Infect Dis. 2006;12(3):489-491. doi:10.3201/eid1205.050560

16. Bortolaia V, Kaas RS, Ruppe E, et al. ResFinder 4.0 for predictions of phenotypes from genotypes. J Antimicrob Chemother. 2020;75 (12):3491-3500. doi:10.1093/jac/dkaa345

17. Zeng L, Zhan Z, Hu L, et al. Genetic characterization of a blaVIM24-carrying IncP-7 $\beta$ plasmid p1160-VIM and a blaVIM-4-harboring integrative and conjugative element Tn6413 from clinical pseudomonas aeruginosa. Front Microbiol. 2019;10:213. doi:10.3389/ fmicb.2019.00213

18. Wang MG, Zhang RM, Wang LL, et al. Molecular epidemiology of carbapenemase-producing Escherichia coli from duck farms in south-east coastal China. J Antimicrob Chemother. 2021;76 (2):322-329. doi:10.1093/jac/dkaa433

19. Yang R-S, Feng Y, Lv X-Y, et al. Emergence of NDM-5-and MCR-1-producing Escherichia coli clones ST648 and ST156 from a single muscovy duck (Cairina moschata). Antimicrob Agents Chemother. 2016;60(11):6899-6902. doi:10.1128/AAC.01365-16

20. Mediavilla JR, Patrawalla A, Chen L, et al. Colistin- and carbapenem-resistant Escherichia coli harboring mcr-1 and blaNDM-5, causing a complicated urinary tract infection in a patient from the United States. MBio. 2016;7(4):e01191-16. doi:10.1128/mBio.01191-16

21. Yu H, Qu F, Shan B, et al. Detection of the $m c r-1$ colistin resistance gene in carbapenem-resistant enterobacteriaceae from different hospitals in China. Antimicrob Agents Chemother. 2016;60 (8):5033-5035. doi:10.1128/AAC.00440-16

22. Zhang Y, Liao K, Gao H, et al. Decreased fitness and virulence in ST10 Escherichia coli harboring blaNDM-5 and mcr-1 against a ST4981 strain with blaNDM-5. Front Cell Infect Microbiol. 2017;7:242. doi:10.3389/fcimb.2017.00242

23. Yao X, Doi Y, Zeng L, Lv L, Liu JH. Carbapenem-resistant and colistin-resistant Escherichia coli co-producing NDM-9 and MCR-1. Lancet Infect Dis. 2016;16(3):288-289. doi:10.1016/ S1473-3099(16)00057-8

24. Lin YC, Kuroda M, Suzuki S, Mu JJ. Emergence of an Escherichia coli strain co-harbouring mcr-1 and blaNDM-9 from a urinary tract infection in Taiwan. J Glob Antimicrob Resist. 2019;16:286-290. doi:10.1016/j.jgar.2018.10.003

25. Liu BT, Song FJ. Emergence of two Escherichia coli strains co-harboring mcr-1 and bla NDM in fresh vegetables from China. Infect Drug Resist. 2019;12:2627-2635. doi:10.2147/IDR.S211746

26. Liu BT, Song FJ, Zou M, Hao ZH, Shan H. Emergence of colistin resistance gene mcr-1 in cronobacter sakazakii producing NDM-9 and in Escherichia coli from the same animal. Antimicrob Agents Chemother. 2017;61(2):e01444-16. doi:10.1128/AAC.01444-16

27. Yang J, Wang HH, Lu Y, et al. A ProQ/FinO family protein involved in plasmid copy number control favours fitness of bacteria carrying mcr-1 -bearing IncI2 plasmids. Nucleic Acids Res. 2021;49 (7):3981-3996. doi:10.1093/nar/gkab149

28. Zheng B, Yu X, Xu H, et al. Complete genome sequencing and genomic characterization of two Escherichia coli strains co-producing MCR-1 and NDM-1 from bloodstream infection. Sci Rep. 2017;7(1):17885. doi:10.1038/s41598-017-18273-2

29. Ovejero CM, Delgado-Blas JF, Calero-Caceres W, Muniesa M, Gonzalez-Zorn B. Spread of mcr-1-carrying Enterobacteriaceae in sewage water from Spain. J Antimicrob Chemother. 2017;72:1050-1053.

30. Barbieri NL, Pimenta RL, de Melo DA, Nolan LK, de Souza MMS, Logue CM. Mcr-1 identified in fecal escherichia coli and avian pathogenic E. coli (APEC) from Brazil. Front Microbiol. 2021;12:659613. doi:10.3389/fmicb.2021.659613 
31. Meinersmann RJ, Ladely SR, Plumblee JR, Cook KL, Thacker E. Prevalence of mcr-1 in the cecal contents of food animals in the United States. Antimicrob Agents Chemother. 2017;61(2):e02244-16. doi:10.1128/AAC.02244-16

32. Lai CC, Chuang YC, Chen CC, Tang HJ. Coexistence of MCR-1 and NDM-9 in a clinical carbapenem-resistant Escherichia coli isolate. Int $J$ Antimicrob Agents. 2017;49(4):517-518. doi:10.1016/j. ijantimicag.2017.02.001
33. Luo Q, Wan F, Yu X, et al. MDR Salmonella enterica serovar Typhimurium ST34 carrying mcr-1 isolated from cases of bloodstream and intestinal infection in children in China. J Antimicrob Chemother. 2020;75(1):92-95. doi:10.1093/jac/dkz415

\section{Publish your work in this journal}

Infection and Drug Resistance is an international, peer-reviewed openaccess journal that focuses on the optimal treatment of infection (bacterial, fungal and viral) and the development and institution of preventive strategies to minimize the development and spread of resistance. The journal is specifically concerned with the epidemiology of

Submit your manuscript here: https://www.dovepress.com/infection-and-drug-resistance-journa antibiotic resistance and the mechanisms of resistance development and diffusion in both hospitals and the community. The manuscript management system is completely online and includes a very quick and fair peerreview system, which is all easy to use. Visit http://www.dovepress.com/ testimonials.php to read real quotes from published authors. 